Des babioles, Des bricoles

\title{
Des babioles, des bricoles
}

Et quelques considérations plus sérieuses

knick-knacks and Trifles : an Inroduction

Olivier Gosselain, Renaud Zeebroek et Jean-Michel Decroly

\section{OpenEdition \\ Journals}

Édition électronique

URL : https://journals.openedition.org/tc/4528

DOI : $10.4000 /$ tc. 4528

ISSN : $1952-420 X$

Éditeur

Éditions de l'EHESS

\section{Édition imprimée}

Date de publication : 15 août 2009

Pagination : 10-17

ISBN : 978-2-7351-1235-7

ISSN : 0248-6016

Référence électronique

Olivier Gosselain, Renaud Zeebroek et Jean-Michel Decroly, "Des babioles, des bricoles », Techniques \& Culture [En ligne], 51 | 2009, mis en ligne le 15 juin 2011, consulté le 29 septembre 2022. URL : http:// journals.openedition.org/tc/4528; DOI : https://doi.org/10.4000/tc.4528 


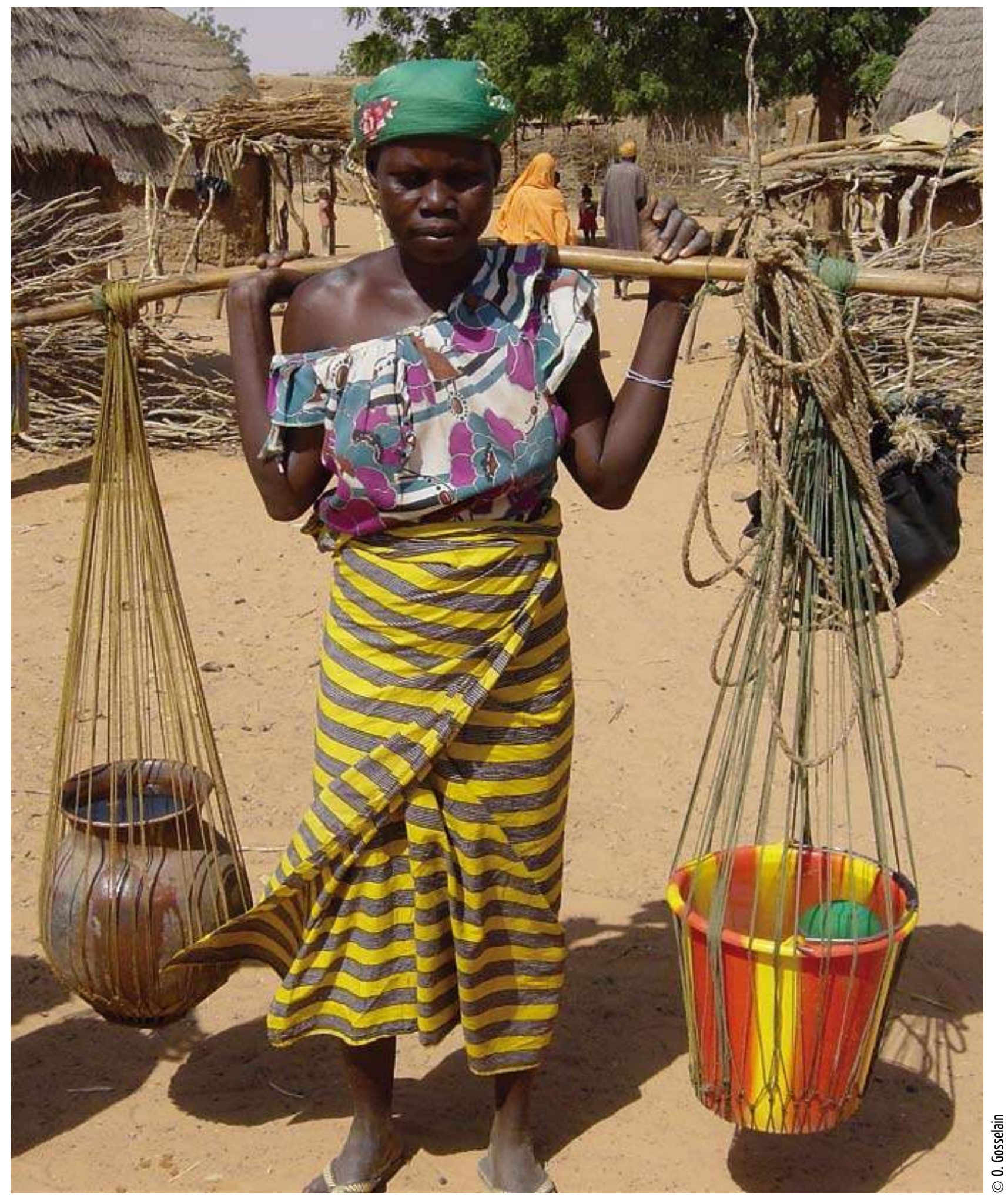




\title{
DES BABIOLES, DES BRICOLES
}

\section{et quelques considérations plus sérieuses}

\author{
"C'est quoi ce machin sur la couverture? Pas les casseroles: le truc "Advisory" \\ là? Ça veut dire quoi? " Les réactions de ce type ont fleuri dès l'élaboration de la \\ maquette de couverture. Une explication s'impose, en introduction du Thema.
}

Faire figurer un sticker de mise en garde - même détourné - sur la couverture d'une publication scientifique ne va pas de soi; surtout lorsque le sticker en question signale habituellement l'usage de grossièretés et d'images explicites dans les disques de rap et de rock. Que les lecteurs se rassurent (ou déchantent) : pas d'obscénités dans ce numéro, du moins au sens général du terme. Juste un détournement d'usage/d'image - il y en a d'autres dans le Thema - pour aborder, sur un mode humoristique, un problème qui est en réalité très académique.

Un mot d'explication s'impose. Le sticker est né en 2003, au retour d'une session de l'American Anthropological Association consacrée à la « transmission culturelle ». L'un de nous avait assisté, abasourdi, à une série d'exposés traitant de la culture comme d'un phénomène totalement désincarné, dont l'évolution était censée obéir aux mêmes lois que celles qui régissent l'évolution du monde naturel. Transmissionniste, utilitariste et adaptationiste, la vision du monde véhiculée dans ces contributions en gommait toute la complexité, toute l'épaisseur historique, pour ne proposer au final que d'insipides modèles de la réalité, sans aucune assise empirique. 


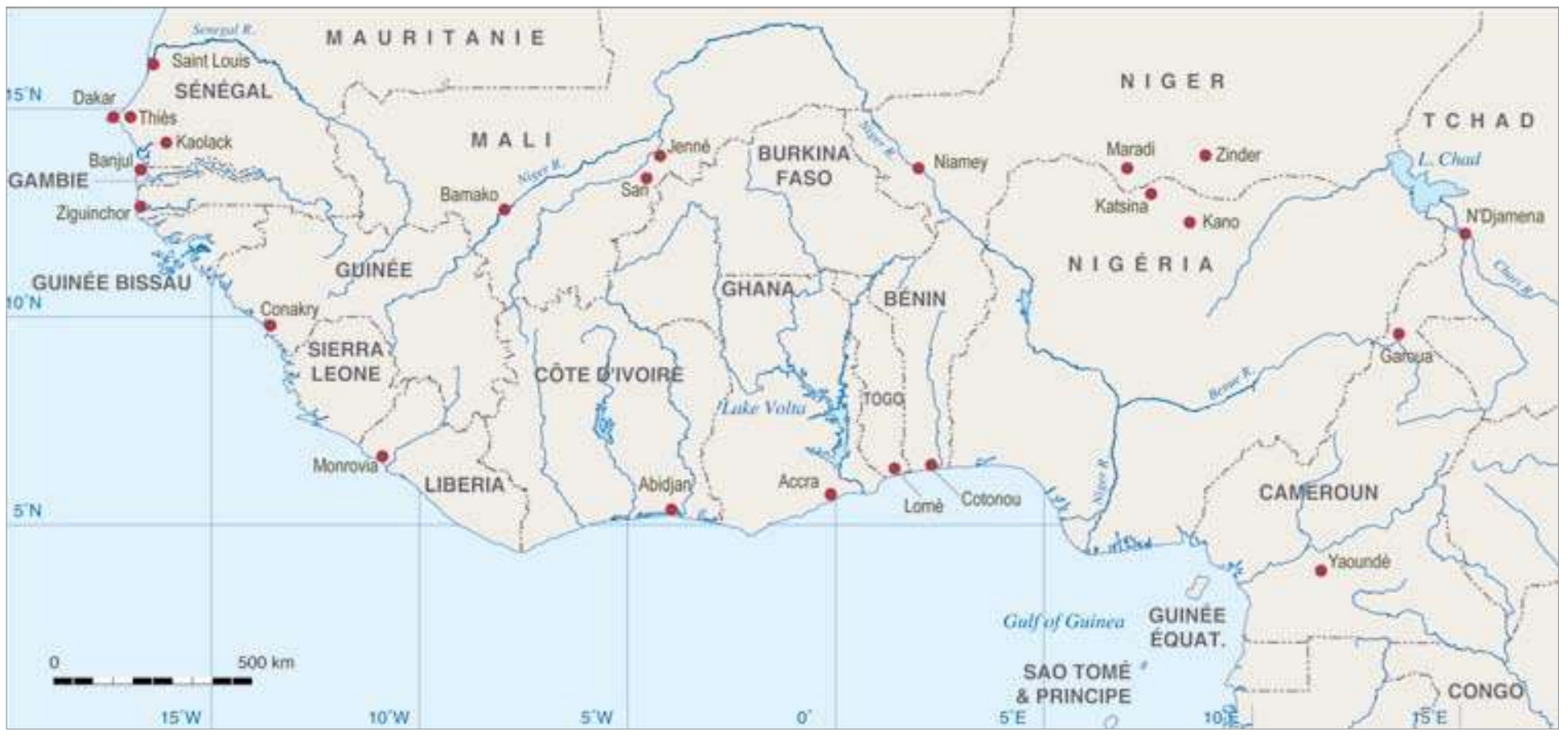

\section{Afrique de l'Ouest et Niger}

Localisations des principaux lieux évoqués dans les contributions du Thema.

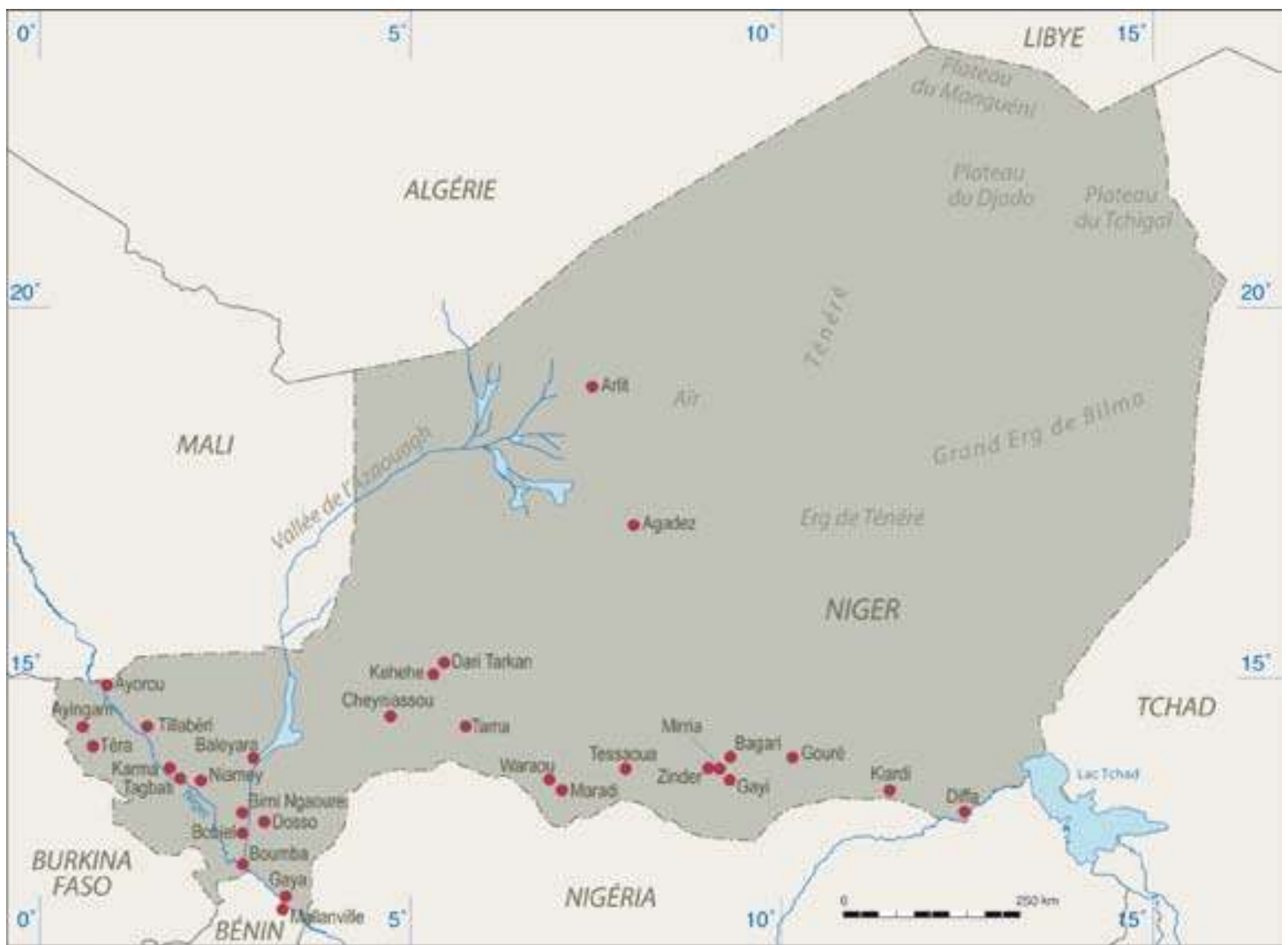


Nous lancions alors le projet pluridisciplinaire « Gestes, objets, lexiques. Analyse multiscalaire de transmissions culturelles » à l'Université libre de Bruxelles ${ }^{1}$, avec pour principale ambition d'étudier d'une nouvelle façon les questions de diffusion et de dynamiques culturelles. Ces problématiques étaient anciennes et un sérieux dépoussiérage s'imposait ; mais fallait-il pour autant succomber aux mirages d'une approche biologisante de la culture? C'est en mesurant le décalage entre les modèles proposés par une telle approche et la complexité des faits observés sur le terrain que nous avons réalisé à quel point il nous faudrait d'abord repenser nos pratiques de recherche, avant même d'explorer ces questions qui justifiaient la mise sur pied du projet.

La nécessité d'homogénéiser le vocabulaire et les approches s'impose avec brutalité lorsque débute un travail qui réunit des disciplines aussi variées que l'anthropologie, l'archéologie, la linguistique et la géographie. Or, à l'origine, et hormis une volonté de travailler ensemble ou un intérêt partagé pour les dimensions spatiales des pratiques culturelles, l'empirisme et la démarche associée à la Grounded Theory constituaient notre principal vocabulaire commun. C'est comme ça qu'est né le sticker «Advisory »; réaction moqueuse à la dérive modélisante et universalisante des approches contemporaines des dynamiques culturelles et détournement d'une icône culturelle - insignifiante pour le plus grand nombre - qui allait devenir un élément fédérateur et emblématique pour notre équipe. Voilà pour la forme. Voyons maintenant pour le fond.

\section{De l'incongruité des faits et du monde en général}

Le monde qui nous entoure est parcouru d'un flux continu d'objets, d'idées et de personnes, la transformation y est constante et paraît même s'accélérer en ce début de XXI siècle. Aucune zone de la planète n'échappe au phénomène, qui interpelle aussi bien le citoyen que les experts et décideurs de toute nature. On parle sans relâche de " globalisation », de « massification », de « complexification », « d'hybridation $» .$. Mais que fait-on concrètement pour en comprendre la nature ou en saisir la portée?

L'une des réponses de nos disciplines a été d'établir une coupure nette entre un « avant» et un «maintenant»- ou pour faire simple, entre « tradition » et «modernité ». Il y aurait d'un côté des objets, des pratiques, des idées, dont l'histoire, forcément longue, serait intrinsèquement liée à celle des peuples qui en font usage; et de l'autre, toutes ces choses « hybrides », " kitch », nées de contacts contemporains entre des univers culturels différents. Personne n'est dupe de la faiblesse d'une telle opposition et peu de scientifiques seraient sans doute prêts aujourd'hui à en défendre la pertinence. Nos pratiques tendent pourtant à la maintenir, voire à la renforcer. Considérons les expériences 
muséales récentes, en France comme ailleurs: la mécanique de légitimation en cours fait que l'objet ethnographique collecté au début du XX siècle et au sujet duquel on ne sait à peu près rien continue à occuper l'avant-scène, paré de la noblesse de l'élément signifiant, esthétique et emblématique d'une culture. Sa version contemporaine, dont la production et l'usage sont parfois bien documentés, est rarement jugée digne d'être montrée au public. Et lorsqu'elle l'est, c'est dans le cadre d'une exposition temporaire dont le dispositif met l'accent sur le caractère ludique, décalé et politiquement critique du discours. Deux régimes de présentation, donc, comme si nous étions en présence de catégories d'objets ontologiquement distinctes.

Du côté des chercheurs, on ne peut pas dire que la "modernité » ne retienne pas l'attention. Elle est même devenue un point focal ces dernières années, au point d'entraîner une reformulation des champs d'étude et des approches, qui se construisent désormais en opposition aux thématiques et méthodes « classiques». Les perspectives sont «transnationales »-concept ironique pour l'essentiel des régions du monde où « l'État Nation » n'est qu'un avatar récent -, les terrains sont «multisitués » et essentiellement urbains, et puis, surtout, l'approche thématique s'est substituée à celle des aires culturelles. Il ne s'agit pas de rejeter cette évolution en bloc: par bien des aspects, elle a permis de recadrer des pratiques de recherche souvent entachées de préjugés et de primitivisme. Mais l'une des dérives actuelles est la superficialité des enquêtes, comme s'il suffisait, pour comprendre les flux contemporains, d'être soi-même perpétuellement en mouvement, de zapper d'un terrain à l'autre, sans se donner vraiment les moyens d'en acquérir une connaissance approfondie. Une fois encore, deux régimes de recherche, comme s'il y avait ici aussi deux catégories de faits sociaux distincts.

Dans ce Thema, l'une de nos volontés est de rappeler avec force l'unicité de l'objet en sciences humaines et sociales. Il s'agit d'aborder, d'étudier et de donner à voir les « choses » du monde dans la même perspective; et peu importe qu'il s'agisse d'une cocotte bariolée ou d'une faience émaillée de Bernard Palissy, de la procession de l'achoura chez les musulmans chiites ou d'un cortège d'halloween en Belgique. Au bout du compte, le seul impératif est de disposer d'un cadre d'analyse unitaire qui permette la comparaison et la compréhension des processus, indépendamment de leur ancrage spatial et temporel. Mais ce cadre ne doit pas se transformer en carcan - nous y reviendrons.

Un aspect crucial dans la mise en œuvre d'un tel projet est le temps. Il faut indéniablement du temps pour échapper à la superficialité d'une approche polarisée: le temps de se confronter au terrain et aux données, le temps de s'en étonner, de comprendre ce que l'on voit et, par-dessus tout, de se tromper, pour pouvoir enfin saisir les enjeux de ce que font les autres et de ce que l'on fait soi-même - comme le démontre magistralement Jean Lave en clôture de ce Thema. 


\section{Lorsque les faits ne sont plus nécessaires}

Un reproche que l'on ne peut certainement pas faire aux modèles néoévolutionistes évoqués plus haut est de polariser les approches. Au contraire, l'unicité et l'universalisme sont ici de rigueur. Toutefois, ces « qualités» ne sont atteintes qu'au prix d'un formidable écrasement de la réalité et d'une simplification outrancière. Comme le savent très bien les chercheurs en sciences exactes, l'élaboration d'un modèle n'est envisageable qu'en isolant le phénomène de l'influence de multiples variables susceptibles d'en infléchir l'évolution. Rien de neuf à cela. Or, on assiste depuis plusieurs années à une dérive "scientiste » dans les sciences humaines et sociales, qui se croient obligées - ou sont forcées - d'emprunter leurs outils aux sciences de la nature, au risque de se déshumaniser. Comme le soulignait, Y.-C. Zarka, dans Libération du 27 juillet 2004, «l'idéologie scientiste, loin de promouvoir un échange plus intense entre les "sciences dures" et les sciences humaines, déséquilibre radicalement leurs rapports en contraignant les secondes à mimer les premières. » Nous voyons actuellement les effets catastrophiques qu'a eu sur l'économie une croyance déraisonnable dans la vertu de modèles mathématiques. Mais le déséquilibre qu'évoque Zarka se traduit aussi par une politique d'évaluation et de financement qui mettent au tapis ceux qui se soucient encore de promouvoir des approches humanistes. Ce n'est un secret pour personne: les plus belles tartuferies en matière de sciences humaines et sociales ont été publiées dans les revues les plus cotées en sciences naturelles, actuellement au sommet de nos systèmes de classement.

Au final, nos disciplines se retrouvent dans une position terriblement inconfortable lorsqu'il s'agit d'expliquer le fonctionnement des sociétés, coincées entre deux formes de simplification : la polarisation et la modélisation.

\section{Que faire?}

Précisons-le d'emblée: notre volonté dans ce Thema n'est pas de proposer un énième modèle d'interprétation. À partir d'études empiriques approfondies, il s'agit plus modestement de mobiliser des outils d'analyse - parfois anciens - issus de différentes disciplines, afin de donner du sens, dans un langage partagé, aux phénomènes que nous étudions. Aucune discipline ne peut avoir la prétention d'expliquer à elle seule les faits sociaux. Il faut nécessairement travailler ensemble, mais non sur base d'une association de circonstance ou d'une juxtaposition de méthodes. Notre façon de faire consiste à puiser dans les répertoires des différentes disciplines pour bricoler des outils utilisables par tous. 
«Bricoler» est ici une notion centrale, qui illustre bien la façon dont nous concevons l'innovation, qu'elle soit scientifique ou technique. En ce sens, « Repenser les dynamiques culturelles » est bien plus une invitation à « penser à nouveau » qu'à endosser un nouveau « prêt à penser ». Mais ici encore, le temps est un facteur crucial. Sans lui, un tel projet est irréalisable.

Au final, notre contribution se veut un point de vue sur les dynamiques culturelles - au sens où Haudricourt parlait de "point de vue humain sur l'objet »dans sa définition de la technologie. Ce point de vue consiste à envisager les dynamiques culturelles en tant que combinaisons de processus qui se déploient à différentes échelles spatiales et suivant des logiques qui leur sont propres. Pour saisir les modalités de transformation ou de rejet d'une nouveauté dans un espace social donné, il s'agit d'envisager à la fois ses caractéristiques propres, les particularités des vecteurs d'introduction et celles du contexte.

Partant des objets et des pratiques, on laisse ainsi une place à la surprise, à de l'indéterminé, ce qui garantit le plaisir et la vitalité de l'analyse. Les différentes contributions de ce Thema l'illustrent bien et s'inscrivent de ce fait dans la ligne éditoriale que n'a cessé de défendre la revue Techniques $\&$ culture. C'est ainsi que nous souhaitons conclure, en revenant sur le fameux sticker de la couverture. Qu'on ne se méprenne pas: il ne s'agit pas d'une mise en garde aux lecteurs et contributeurs habituels de la revue, mais bien d'un hommage pour avoir résolument défendu l'importance du terrain et des faits depuis plus de trente ans, indépendamment des modes et des pressions académiques.

\section{NOTES}

1. Ce projet a été financé par les « Actions de Recherche Concertée » de la DGENORS (Direction de la Recherche scientifique de la Communauté française de Belgique). 


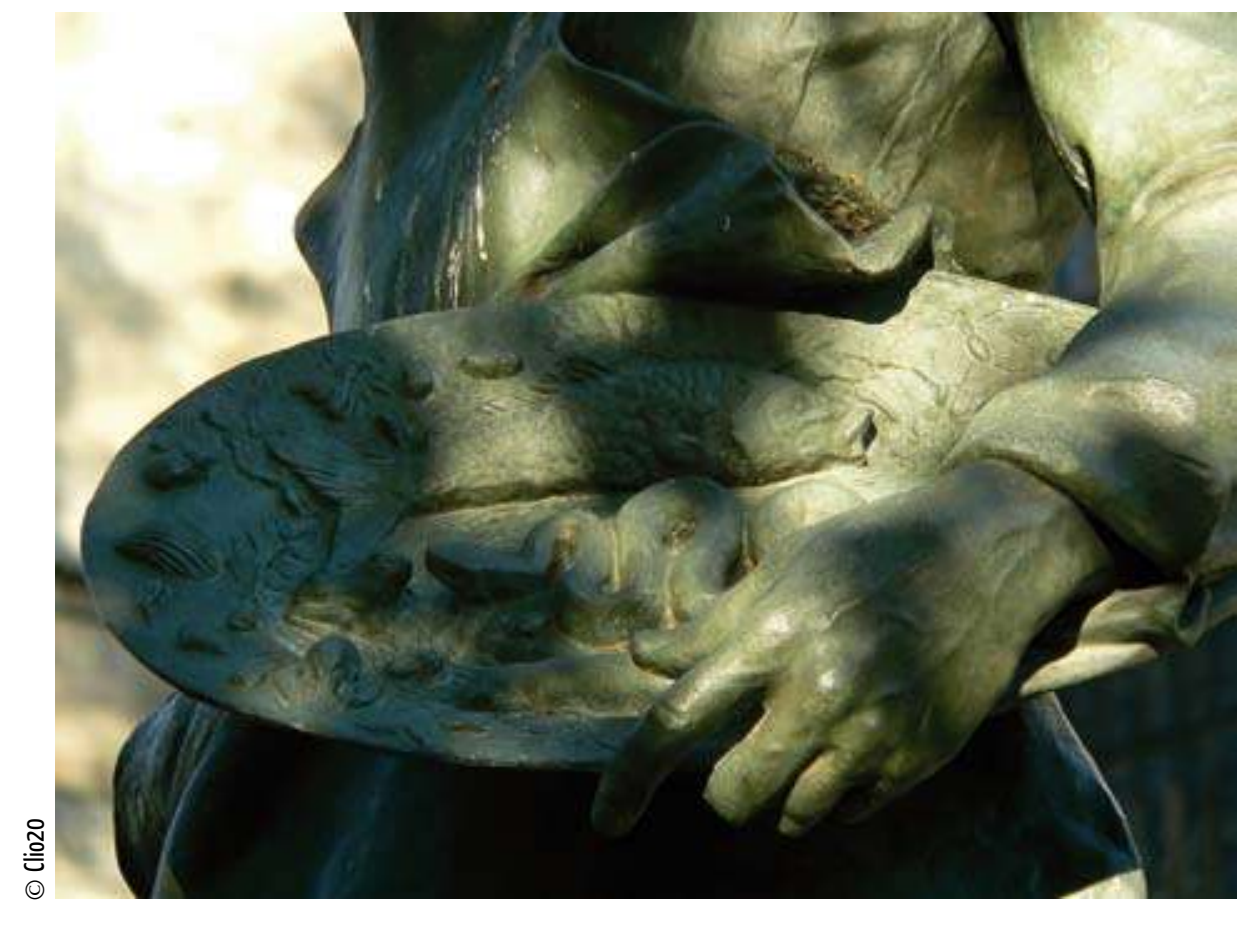

Statue de Bernard Palissy (c. 1510-1589)

à Saint-Germain-des-Prés, Paris (Détail). 